structure, and arrived at the expectation of a maximum relaxation time at the transition midpoint - which, the indications are, is nevertheless still in the submicrosecond range.

The general accord on the characteristics of the transition was breached only by the unexpected observation that proton magnetic resonance was able in certain cases to resolve contributions from $\alpha$-carbon proteins in the $\alpha$-helical and the randomly coiled states. Now this would imply slow interconversion between these conformations relative to the nuclear relaxation time scale, in other words a transition rate which is a round half-dozen orders of magnitude slower than it should be. Various explanations were offered for this phenomenon, of which only two survived closer scrutiny. One was that the polypeptide samples are all polydisperse, and because the longest chains form the most stable helices, and exhibit the sharpest transitions, one is simply resolving the signals from different size populations. The other suggestion was that the helix-nucleation step is slow in the polymer-solvent combinations in question, and the distribution of conformations at equilibrium is in fact very different from that calculated from the Ising lattice model.

This proposition has now been reexamined by Miller (Macromolecules, 6 , 100 ; 1973), who has calculated conformational lifetimes for more elaborate models. In particular he has examined the effect of the assumption, made for ease of computation, that the probability of finding more than one run of helix within a single chain is low. This he shows leads to an overestimate of the helix lifetime. The values for the lifetimes also depend on the balance of the thermodynamic parameters for helix formation and the chain length. By choosing a combination of values for the parameters in the model, Miller is able to simulate the observed NMR spectra. That the splitting of helix and coil contributions has been observed only in non-aqueous solvent systems can be easily enough rationalized on the basis of different values for the helix initiation parameter in the two cases.

It only partly detracts from the interest of this analysis that the NMR results which it seeks to explain are evidently in fact no more than a consequence of polydispersity. In the interim Nagayama and Wada (Chem. Phys. Lett., 16, 50 ; 1972) have examined very narrow cuts of polybenzylglutamate (with a weight to number average molecular weight ratio of 1.04), and shown that there is no $\alpha$-carbon proton splitting in the helix-coil transition region, thereby, one may hope, laying the ghost once for all.

One of the few approaches to rates of conformational transitions in these short time ranges is the measurement of ultrasonic absorption. In aqueous polyglutamic acid a relaxation corresponding to the helix-random coil transition was observed, and showed a maximum at the $p \mathbf{H}$ of the transition midpoint, as demanded by Schwarz's theory. Other ultrasonic absorption results, however, were too hastily interpreted and proton transfer processes were wrongly taken for the helix-coil transition. Zana and Lang (Biopolymers, 12, 79 ; 1973) have now done measurements on polybenzylaspartate in a mixed solvent system, and find that though the level of absorption is different in the $\alpha$-helical and random states-presumably in consequence of solvation differences-no maximum of absorption, such as would correspond to the transition process itself, is to be observed in the working frequency range. This makes it possible to set a lower limit on the relaxation time of the transition. The corresponding rate constant is higher by at least an order of magnitude than that observed for polyglutamic acid in water.

The various limitations in all these methods should direct all the more attention to an entirely new and potentially very versatile approach to fast kinetics. Feher and Weissman (Proc. US Nat. Acad. Sci., 70, $870 ; 1973$ ) have evolved a technique for the study of the concentration fluctuations which prevail in a reacting system at equilibrium. The beauty of this method is that the equilibrium is not disturbed in the course of observation. It has been tried out on a system in ionic dissociation equilibrium, using the solution conductivity as the vehicle of observation. Feher and Weissman have succeeded in separating true concentration fluctuation noise from electronic and other types of noise, and have extracted a frequency spectrum for the fluctuation. The temperature dependence yields a linear Arrhenius plot, and an activation energy agreeing precisely with the value from relaxation measurements. Inasmuch as conformational processes involving macroions, such as nucleic acids and proteins, are in general accompanied by changes in counterion binding and therefore solution conductivity, this approach may well be one of the brightest prospects now on the horizon.

\section{INFLUENZA VIRUS}

\section{Human Pandemic Strains}

from our Cell Biology Correspondent

IN 1968 the so-called Hong Kong strain of influenza virus arose in China and proceeded to cause a worldwide epidemic of influenza. The origin of Hong Kong influenza virus has remained something of a mystery, but two virologists, Laver and Webster, who have a bent towards molecular detective work, believe they may at last have uncovered the origin of this virulent virus (Virology, 51, 383 ; 1973).

Last year Webster and Laver reported that the haemagglutinin subunits of the 1968 Hong Kong virus and preceding A2 Asian influenza viruses were completely different both in their immunology and their amino acid sequence. These observations made it highly unlikely that the Hong Kong virus was simply a mutant of some pre-existing human influenza virus. On the other hand, in 1968 and 1969 others had shown that the neuraminidase subunits of the Hong Kong virus were antigenically identical to those of preceding A2 Asian influenza viruses. These two

\title{
Sequencing of Sticky Ends
}

MANY coliphages, the most familiar example being lambda phage, contain a double stranded genomic DNA molecule with what are known as cohesive or sticky ends. The two ends of such molecules are single stranded and mutually complementary in base sequence so that the linear molecules can form circles, the two single stranded tails base pairing with each other, or can polymerize to form a linear or circular concatamers. The base sequences of the sticky ends of the lambdoid phages are apparently similar because DNA of phages of this family form mixed dimers, the single stranded tail of one phage DNA base pairing with that of its relative. Likewise another family of temperate coliphages, which include phages 186, P2 and 299, show a similar relationship with each other but not with the lambdoid phages. Murray and Murray have directly con- firmed these relationships by analysing the base sequences of the sticky ends of five lambdoid phages and three other temperate phages. They report their results in Nature New Biology next Wednesday (May 30).

Their painstaking analysis, which testifies to the considerable progress that is being made in the development of techniques for sequencing directly DNA molecules, proves that the cohesive ends of the five lambdoid phages studied have identical nucleotide sequences. The sequences of the sticky ends of phages 186, P2 and 299 by contrast are not identical but they are similar, and sufficiently so for the DNAs to cohere in spite of regions of mismatch. These data indicate that during the evolution of these phages the sequence of the sticky ends has been conserved no doubt because of the part they play in the life cycle of the phages. 